\title{
A Web Based History tool for Multicast e-Meeting Sessions
}

\author{
Roland Parviainen and Peter Parnes \\ Division of Media Technology \\ Department of Computer Science and Electrical Engineering \\ Luleå University of Technology \\ 97187 Luleå, Sweden \\ Roland.Parviainen@ltu.se,Peter.Parnes@ltu.se
}

\begin{abstract}
This paper presents a web based history tool for multicast based e-meeting systems. The tool allow users to see past events and activity graphs of current and past sessions. Video snapshots are stored when events are reported, making it possible to quickly get an overview of the history of a session. This tool has several uses such as a quick and easily accessible way to improve group awareness, monitoring and review of meetings and lectures.
\end{abstract}

\section{Introduction}

Video conferencing and e-meetings systems are today commonplace on the Internet in several environments, especially in businesses that want to save money on travel costs or time spent on attending physical meetings. These systems are used for meetings, presentations and to continuously support for collaboration in a group setting, providing among other features text messaging, audio and video, and shared collaboration tools and provides a sense of group awareness to members. Tools for reviewing the history of an e-meeting session have several uses:

Group awareness: If a user is not able to participate in a session, the history tool can be used to get a feeling of group awareness by enabling the user to get a quick overview of the history of a session and see who has been active at what times.

Reviewing meetings or lectures: To review meetings or lectures after they have occurred, for example to see which students where active during the lecture can be very important. If the actual content, e.g. what was said, viewing a playback of a recorded meeting would of course be better.

Monitoring and surveillance: For these uses it is very important the system really stores all important video snapshots, i.e. every time something changes in a video stream.

Research: A history tool is invaluable when doing research about the use of e-meeting tools themselves.

Several tools exist to record video conferences and e- meeting sessions, but there are some inherent problems: a need to remember to start the recording, the recording takes up a large amount of disk space and a viewing a recording of a session can take a long time and can be cumbersome. To record continuous sessions that run 24 hours a day is not practical. Tools that create summaries of recorded sessions or video are also common[2][4], but while these tools can make it much easier to get a quick overview of a session they still need a complete recording of the session. The history tool described in this paper only store a minimum of information needed; the summaries are created at real time during the session and stored in a database for easy and flexible access to information.

As the storage of video snapshots and other session information can be sensitive the privacy issues have to be considered carefully. In this tool, users can themselves set what information the tool is allowed to use and store. The access to the tool is restricted through basic HTTP authentication and optionally protected through TLS/SSL encryption.

\section{Background}

At Luleå University of Technology collaborative workspaces are used daily in a wide variety of situations. Example uses include allowing students to view classroom lectures from home, enabling members of discussion groups to interact from a distance and providing members of projects and research divisions with increased presence of each other throughout their work day. The last mentioned case is referred to as the "e-corridor".

In the e-corridor members of the session can be either active or passive and the possible uses of the workspace range from giving a formal presentation to passive monitoring of video. Communicating with colleagues through the e-corridor often replaces other communication media such as e-mail, phone calls or personal visits. Users with broadband Internet access at home can choose to be part of the e-corridor, participate in meetings and follow presentations.

The software used for the e-corridor is Marratech Pro, a 

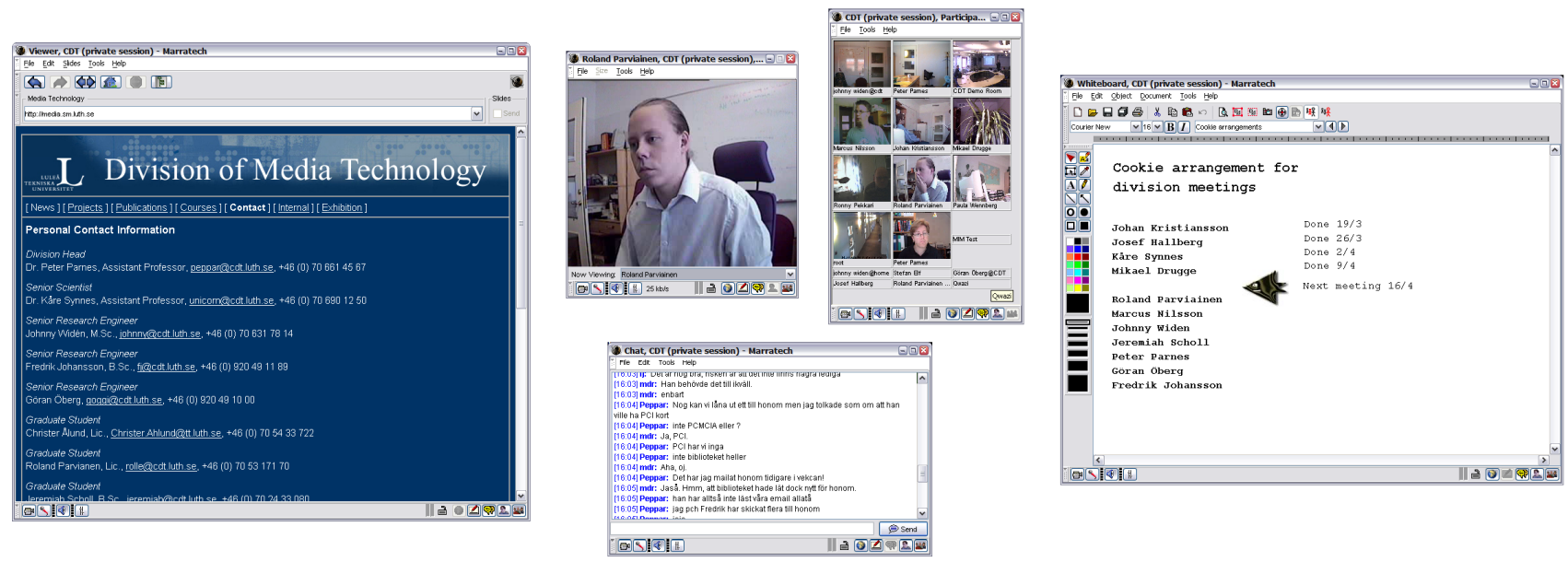

Figure 1. Screen-shot of the different tools of Marratech Pro

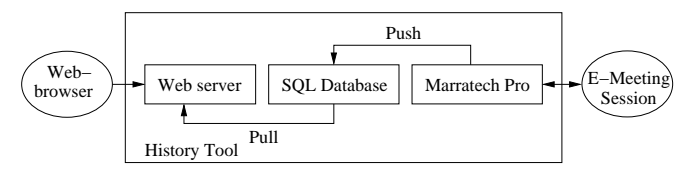

Figure 2. Architecture

commercial application by Marratech $\mathrm{AB}^{1}$, which is based on earlier research done by the Media Technology research group[5]. Marratech Pro provides the users with the ability to send and receive audio and video to and from other participants. In addition there are a shared whiteboard and a shared web browser. The chat, shared whiteboard and shared web browser all use proprietary protocols while the audio and video tools use standard protocols such as RTP. Marratech Pro can use either IP multicast or unicast. In the latter case traffic is tunneled through a media gateway called the "E-Meeting Portal". The video streams from participants in an e-meeting are presented in the "participants" window, which gives a thumbnail overview of all the video streams currently received from the group, while a "focus" window displays the video obtained from a single group member with a higher resolution and frame rate. All audio streams that are not manually muted by the user are mixed and played synchronized to the video streams. The shared whiteboard is mainly used to make simple drawings, application sharing and sharing Microsoft PowerPoint slides during presentations.

A web interface to Marratech Pro also exist[6]. It is mainly used for accessing the e-corridor when the regular application is not available or not usable. It only uses features available in HTML 4.01, except for audio where it requires a MP3 player that supports HTTP streaming. During

\footnotetext{
${ }^{1}$ http://www.marratech.com
}

the use of the Marratech Pro web interface, it became clear that it would be very useful to be able to see events in past as well, not just what is currently happening.

\section{Architecture}

The Marratech Pro application have been modified to process and send events and data from the currently joined session to an SQL database, from where a web interface retrieves and present information. See figure 2 for a description of the architecture. A web front end matches our needs and uses, such as good availability and the ability to get a quick overview of past events and a sense group awareness. Other uses such as analysis of tool use and statistics can use generic SQL query tools. The modified Marratech Pro can either be used as a non active participant in a session whose only task is to receive information or completely replacing the standard version or act as a personal history tool for a single user, recording events for all sessions the user participates in.

\subsection{Processing of different media}

While recording of the video streams of a session provides the complete video history, the storage needs can quickly grow beyond available resources. Only storing snapshots at regular intervals still may result in requiring to much storage space, while this also can miss important events if the interval is too large. Both methods stores large amount of redundancy: for example, in the e-corridor, during night time when there usually is no activity it is unnecessary to store any video information. It can also be difficult to find interesting events. An alternative method which is used in the system presented in this paper is to store snapshots when activity is detected in the video streams. To make sure the event that generated the video activity is represented in 


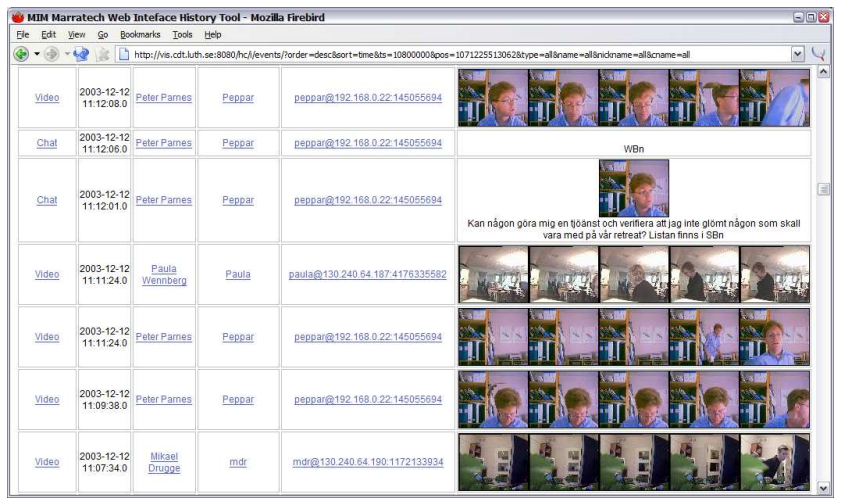

Figure 3. Showing events with snapshots

the history snapshots are taken before and after activity are detected as well. The algorithm is described in more detail in section 4.

When a chat message is received, the text of the message, the sending user and a timestamp is stored in the database and if available, a current video snapshot is also stored. Information about private chat messages between users are not stored. Similarly, when a web page is distributed through the shared web browser the URL is stored along with the sending user, a timestamp and a video snapshot. Audio and whiteboard activity is directly reported as events, with a maximum of 6 events per minute per media. Mixed audio data can optionally be stored in the database, in the form of 16Kbit/s MP3 data.

\subsection{Web front-end}

The web front-end retrieves and presents the data from the database. Two types of displays are available, a list of events with snapshots and a list of activity indicators for each user:

Events with snapshots: The event list shows event type, timestamp, user, snapshot and event data if applicable, i.e. the event is a chat message or a URL. Users can select and sort events by user name, event type and timestamp. The time span to show on one page can also be selected. See figure 3 for an example.

Activity indicators: The activity indicator list shows activity indicators for each user which displays different activities in different colors. Each activity indicator shows either one hour, one day or one week of activity. Figure 4 shows an example of the activity of one day.

In both views it is possible to retrieve any audio from the selected time interval as a HTTP stream.

\subsection{Activity indicators}

The purpose of the activity indicators is to, in a simple way, get a quick overview of all events in a longer time span

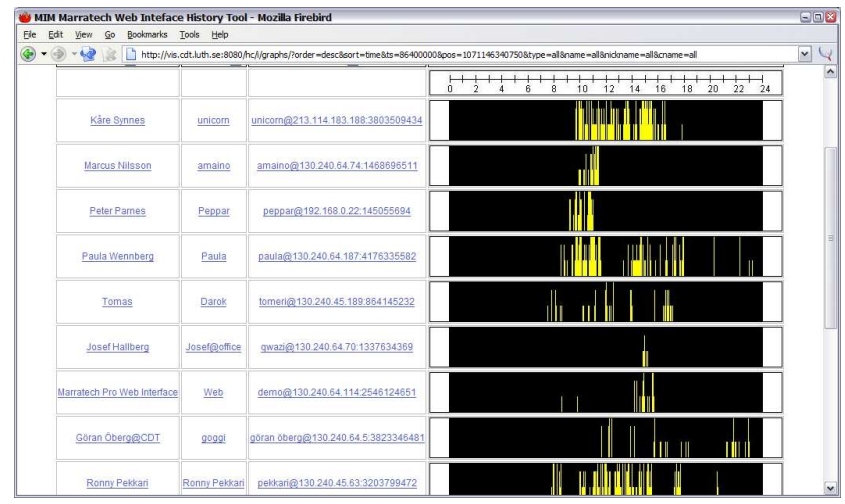

Figure 4. Activity indicators

that is possible with viewing video snapshots. Activity indicators are created from the data stored in the database when requested. The indicator graph is divided into bins corresponding to time intervals, and as events are added to the graph they are added to the bin matching the event timestamp. The sum of the values of each bin is drawn in the color representing the highest prioritized media type in the bin. The order of priority is as follows: video have lowest priority, then audio, whiteboard, chat and finally shared web browser events have highest priority. This ensures that e.g. the display of irregular and important events such as chat events are not drowned out by events that can occur often such as audio and video events.

\subsection{User settings}

To maintain users' privacy the history tool can filter out different type of events based on user preferences. These preferences are set through private chat messages to the history tool which are parsed and handled. Three messages are supported. First, !deny [what], where what can either be a list of media types or "all", specifies what media the user does not want to be stored or processed. Similarly, !allow [what] specifies what media are allowed to be stored or processed. By default, all media are allowed. Finally, the !show message returns the current settings. The users are identified by the for the session unique RTCP CNAME parameter. Web based preferences was not included in the tool since it is difficult to map users in the session to users of the web based tool as the user parameters in a session can be chosen arbitrarily by the user and also depends on what computer or network the user is currently using.

\section{Implementation}

Event processing and database insertion part is implemented in Java inside the Marratech Pro application. Events are forwarded from various parts of the application to the history subsystem, which processes event and stores video 
snapshots in an internal cache in a resolution of 96x72 pixels. As the video change detection algorithm detects video events, the five snapshot in the cache with a timestamp closest to the event timestamp are retrieved from the cache and encoded into JPEG images. The handling of video events are delayed so that snapshots both in the past and in the future relative to the timestamps can be used.

Video activity is detected using the following algorithm, similar to the one used in NYNEX Portholes[3]: the sum of the differences in luminance for each pixel between two consecutive frames is used as an activity value. If the difference between the current value and the average of the previous ten values are greater than a specified threshold a video event is reported.

Decoded and mixed audio is encoded as MP3 frames and are stored in the database together with a timestamp.

All HTTP requests to the web front end are handled in a similar way: the request parameters such as time span and sort order are parsed, a SQL query is formed and executed and the query results are formatted for HTML output or binary output.

The web front end is implemented as a Java Servlet, running under the Apache Tomcat 4.1 Servlet container, communicating with the database through JDBC. The MySQL database server is currently used the database management system.

\section{Related Work}

In [1] Chen describes a system for visualizing the activity in online lectures which includes a similar view as the activity graph view in our system, but a view comparable to the event view is not available. One method for creating summaries for recorded meetings is described in [2]. We employ similar algorithms in realtime in an on-going session to create the history directly. In [4] a system for browsing the history of conferences where spoken language is the main interaction mode is introduced, with some similar features.

\section{Evaluation and future work}

All performance measures in this section were done with the database and servlet engine running on a $2 \mathrm{GHz}$ PC running Windows XP. The tests were run with data from one specific day ( 24 hours) with a total of 13 users, 1832 events and 8983 snapshots. The complete dataset spanned a full month, with more than 30000 events and 135000 snapshots. The average snapshot size was 2430 bytes, while the average size of the activity graphs was 2350 bytes. Downloading the activity graphs view (HTML and all images, both dynamically generated) takes $1.1 \mathrm{~s}$ with the download tool wget over a 10Mbit/s ethernet connection. Downloading the event list with snapshots takes between 3 and 6 minutes, depending on the tool used. The wide variation in time is due to differences in the number of concurrent download threads and efficiancy in rendering. The HTML page was generated and downloaded in $5 \mathrm{~s}$, the rest of the time was spent downloading the snapshot images. The total size of the page and images was 27.8 Mbyte.

Only informal studies of the usefulness of the tool have been done through interviews with users. The most requested feature was a search function, which will be implemented in the future. While privacy issues have not been a problem for users used to the concept of the e-corridor, other users have raised concerns over the possibility of the history tool being used as a surveillance tool. Researchers in other institutions have also shown interest in using the tool to study the use of Marratech Pro in a work group.

While the algorithm for detecting changes and choosing snapshots works adequately in most instances a better algorithm is needed for optimal performance.

While it is possible to replay audio, it can be hard to find a particular conversation. One possible way to help searching for spoken communication is to use speech recognition. One problem with speech recognition for our use is that the language spoken in session varies, sometimes even several languages are spoken during one conversation.

\section{Conclusion}

We have a presented a web based history tool which captures the history of a multicast e-meeting session. By storing events and video snapshots around critical moments in a database and providing a web interface to the data, a user can easily and quickly get a good overview of the history of a session from a web browser. Audio from selected time intervals can be streamed or downloaded as MP3 data over HTTP. The use of a database also makes it possible to easily calculate and present different statistics of the use of different media and tools in a session.

\section{References}

[1] M. Chen. Visualizing the Pulse of a Classroom. In Proceedings of ACM Multimedia 2003.

[2] B. Erol, D. Lee, and J. Hull. Multimodal summarization of meeting recordings. In Proceedings of the IEEE International Conference on Multimedia \& Expo (ICME 2003).

[3] A. Lee, K. Schlueter, and A. Girgensohn. Sensing activity in video images. In CHI 97 Extended Abstracts. ACM Press, 1997.

[4] S. Luz and D. Roy. Meeting browser: A system for visualising and accessing audio in multicast meetings. In Proceedings of the IEEE Workshop on Multimedia Signal Processing, 1999.

[5] P. Parnes, K. Synnes, and D. Schefström. mStar: Enabling collaborative applications on the Internet. Internet Computing, 4, 2000.

[6] R. Parviainen and P. Parnes. The MIM Web Gateway to IP Multicast E-Meetings. In Proceedings of the SPIE/ACM Multimedia Computing and Networking Conference (MMCN'04), 2004. 